\title{
EXTENSION OF INVARIANT LINEAR FUNCTIONALS: A SEQUEL TO FAN'S PAPER
}

\author{
ANTHONY TO-MING LAU ${ }^{1}$
}

\begin{abstract}
We establish the relationship between certain invariant extension properties for linear functionals of $\mathrm{K}$. Fan when a semigroup $S$ acts on a locally convex topological vector space and the existence of a left-invariant mean on the space of almost periodic and weakly almost periodic functions on $S$.
\end{abstract}

1. Introduction. Recently Fan [1] (see also [2]) showed that when a group $S$ acts on a real Hausdorff locally convex topological vector space $E$ such that for each $x \in E$ the orbit $S(x)=\{s \cdot x, s \in S\}$ is (weakly) relatively compact, then it has certain extension properties for invariant linear functionals. He also pointed out in [1] and [2] that Theorem 3 in [2] (and therefore Theorem 1 in [1]) implies von Neumann's mean value theorem for almost periodic functions. It is the purpose of the present note to clarify more fully this relationship between the extension properties of $S$ for invariant linear functionals and the existence of a left-invariant mean on the space of (weakly) almost periodic functions on $S$. Our approach provides a unified and different proof to both Theorems 1 and 3 in [1]. It also shows that the completeness hypothesis in Theorem 3 of [1] can be removed.

It is our pleasure to thank Professor Ky Fan for providing us with a preprint of his work [1].

Throughout this paper, $S$ will denote a topological semigroup. By a continuous (resp. weakly continuous) right action of $S$ on a topological vector space $(E, \mathscr{T})$ we shall mean a map $S \times E \rightarrow E$ denoted by $(s, x) \rightarrow s \cdot x$ such that

(1) $(a b) \cdot x=b \cdot(a \cdot x)$ for each $a, b \in S$ and $x \in X$.

(2) For each $s \in S$, the map $x \rightarrow s \cdot x$ is a continuous linear map from $(E, \mathcal{T})$ into $(E, \mathcal{T})$.

Received by the editors October 12, 1976.

AMS (MOS) subject classifications (1970). Primary 43A07, 43A60, 22A20.

Key words and phrases. Semigroups, groups, invariant mean, almost periodic functions, extension of invariant functionals.

${ }^{1}$ This research was supported by NRC Grant A-7679. It was conducted while the author was visiting the Department of Mathematics, University of California at Berkeley. We would like to thank members of the department for their kind hospitality during our visits. 
(3) For each $x \in X$, the map $s \rightarrow s \cdot x$ is continuous from $S$ into $(E, \mathcal{T})$ (resp. $(E$, weak)).

The action of $S$ on $E$ is almost periodic (resp. weakly almost periodic) if for each $x \in E$, the orbit $S(x)$ is relatively compact in the $\mathcal{T}$ topology (resp. weak topology).

Let $C(S)$ denote the Banach space of bounded continuous real-valued functions on $S$ with the supremum norm. For each $a \in S, f \in C(S)$, let $\left(l_{a} f\right)(s)=f(a s)$ for each $s \in S$. Let $A P(S)$ (resp. WAP(S)) denote the closed subspace of $C(S)$ consisting of all $f \in C(S)$ such that $\left\{l_{a} f ; a \in S\right\}$ is relatively compact in the norm topology (resp. weak topology) of $C(S)$. A linear functional $\phi$ on $A P(S)$ (or $W A P(S)$ ) is a left-invariant mean if $\|\phi\|=\phi(1)=1$ and $l_{a}^{*} \phi=\phi$ for each $a \in S$, where $l_{a}^{*}$ denotes the transpose of $l_{a}$.

\section{The mean theorems.}

THEOREM 1. The following conditions on a topological semigroup $S$ are equivalent:

(a) $A P(S)$ has a left-invariant mean.

(b) For any almost periodic continuous right action of $S$ on a Hausdorff real topological vector space $E$, if $L$ is an invariant subspace of $E$ and $K$ is a convex subset of $E$ such that $K-x_{0}$ is invariant for some $x_{0}$ contained in $L \cap$ Int $K$, then for each invariant linear functional $\phi$ on $L$ such that $\phi(x) \leqslant \alpha$ for all $x \in L \cap K$ and some fixed real number $\alpha$, then there exists an invariant linear extension $\tilde{\phi}$ of $\phi$ to $E$ such that $\tilde{\phi}(x) \leqslant \alpha$ for all $x \in K$.

Proof. (a) implies (b). We may assume that $\phi$ is nonzero. In this case $\phi\left(x_{0}\right)<\alpha$. As in the proof of Theorem 2 [1], define $\phi_{0}$ on $L$ :

$$
\phi_{0}(x)=\phi(x) /\left(\alpha-\phi\left(x_{0}\right)\right)
$$

and let $K_{0}=K-x_{0}$. Then $\phi_{0}(x) \leqslant 1$ for all $x \in L \cap K_{0}$. Let $p$ be the Minkowski functional on $E$ defined by $K_{0}$ i.e. $p(x)=\inf \left\{\lambda>0 ; x \in \lambda K_{0}\right\}$. Then $p$ is sublinear, $p(s \cdot x) \leqslant p(x)$ for all $s \in S$ and $x \in E$, and $\phi_{0} \leqslant p \mid L$. By the Hahn Banach extension theorem, $\phi_{0}$ has an extension $\eta$ to $E$ such that $\eta \leqslant p$. For each $x \in E$, define $\left(T_{x} \eta\right)(s)=\eta(s \cdot x)$. Then clearly $T_{x} \eta \in C(S)$. Observe that if $x, y \in E$ and $z=x-y$, then $T_{x} \eta(s)-T_{y} \eta(s)=\eta(s \cdot z)$ $\leqslant p(s \cdot z) \leqslant p(z)$ for each $s \in S$. Hence $\left\|T_{x} \eta-T_{y} \eta\right\| \leqslant \max \{|p(z)|,|p(-z)|\}$. Consequently the map $x \rightarrow T_{x} \eta$ is continuous from $E$ into $C(S)$ with the sup norm topology. Also since $l_{a}\left(T_{x} \eta\right)=T_{a \cdot x} \eta$, it follows that $T_{x} \eta$ is almost periodic.

Let $m$ be a left-invariant mean on $A P(S)$. Define $\psi_{0}(x)=m\left(T_{x} \eta\right)$. Then $\psi_{0}$ is an invariant extension of $\phi_{0}$. For $x \in L$ and $s \in S$, we have $\left(T_{x} \eta\right)(s)$ 
$=\eta(s \cdot x)=\phi_{0}(s \cdot x)=\phi_{0}(x)$. So for each $x \in L, T_{x} \eta \in C(S)$ is the constant function $\phi_{0}(x)$, and therefore $\psi_{0}(x)=\phi_{0}(x)$. Also $\psi_{0} \leqslant p$. Consequently $\psi_{0}(x) \leqslant 1$ for all $x \in K_{0}$. Now put $\tilde{\phi}=\left(\alpha-\phi\left(x_{0}\right)\right) \psi_{0}$. Then $\tilde{\phi}$ is the required extension.

(b) implies (a). Consider the continuous right action of $S$ on $A P(S)$ defined by $(a, f) \rightarrow l_{a} f$. Let $L$ be the subspace of $A P(S)$ consisting of constant functions, let $K$ be the unit ball in $A P(S)$ and $\alpha=1$. Let $a \in S$ be fixed, and define $\phi(f)=f(a)$ for each $f \in L$. Then $\phi$ is clearly invariant and $\phi(f) \leqslant 1$ for all $f \in L \cap K$. It is easy to see that any invariant extension $\tilde{\phi}$ of $\phi$ to $A P(S)$ such that $\tilde{\phi}(f) \leqslant 1$ for all $f \in K$ is a left-invariant mean on $A P(S)$.

A simple modification of the above argument also proves:

THEOREM 2. The following conditions on a topological semigroup $S$ are equivalent:

(a) WAP(S) has a left-invariant mean.

(b) For any weakly almost periodic weakly continuous right action of $S$ on a Hausdorff real topological vector space $E$, if $L$ is an invariant subspace of $E$ and $K$ is a convex subset of $E$ such that $K-x_{0}$ is invariant for some $x_{0}$ contained in $L \cap$ Int $K$, then for each invariant linear functional $\phi$ on $L$ such that $\phi(x) \leqslant \alpha$ for all $x \in L \cap K$ and some fixed real number $\alpha$, then there exists an invariant linear extension $\tilde{\phi}$ of $\phi$ to $E$ such that $\tilde{\phi}(x) \leqslant \alpha$ for all $x \in K$.

Remarks. (1) Since $A P(S)$ and $W A P(S)$ always have a left-invariant mean for any group $S$ (see [3, p. 38]) our Theorem 1 implies Theorem 1 in [1] and our Theorem 2 implies Theorem 3 in [1] without completeness hypothesis.

(2) If $a S \cap b S$ is nonempty for any $a, b \in S$, then $S$ has property (b) of Theorem 1, since in this case $A P(S)$ has a left-invariant mean (see [4]).

(3) We have actually proved implicitly the following analogue of Theorem 15.A in Silverman [5]:

THEOREM 3. The following conditions on $S$ are equivalent:

(a) $A P(S)$ has a left-invariant mean.

(b) For any almost periodic continuous right action of $S$ on a Hausdorff real topological vector space $E$, if $p$ is a continuous sublinear functional on $E$ such that $p(s \cdot x) \leqslant p(x)$ for each $s \in S$ and $x \in E$, and if $\phi$ is an invariant linear functional defined on a subspace $F$ of $E$ such that $\phi \leqslant p$, then there exists an invariant linear extension $\tilde{\phi}$ of $\phi$ to $E$ such that $\tilde{\phi} \leqslant p$.

A similar statement for $W A P(S)$ with "almost periodic continuous right action" replaced by "weakly almost periodic weakly continuous right action" also holds. 


\section{REFERENCES}

1. K. Fan, Extension of invariant linear functionals, Proc. Amer. Math. Soc. (to appear).

2. - Orbits of semi-groups of contractions and groups of isometries, Abh. Math. Sem. Univ. Hamburg 45 (1976), 245-250.

3. F. P. Greenleaf, Invariant means on topological groups and their applications, Van Nostrand Math. Studies No. 16, Van Nostrand Rheinhold, Princeton, N.J., 1969. MR 40 \#4776.

4. R. D. Holmes and A. Lau, Non-expansive actions of topological semigroups and fixed points, J. London Math. Soc. (2) 5 (1972), 330-336. MR 47 \# 2447.

5. R. J. Silverman, Means on semigroups and the Hahn-Banach extension property, Trans. Amer. Math. Soc. 83 (1956), 222-237. MR 18, 910.

Department of Mathematics, University of Alberta, Edmonton, Alberta, Canada 